\title{
Atropisomeric Separation of PCB-95 by HPLC
}

\author{
Prabha Ranasinghe ${ }^{1,2}$, Christopher Olivares ${ }^{2}$, William Champion $\mathrm{Jr}^{3}$, Cindy Lee ${ }^{1,2, *}$ \\ ${ }^{1}$ Environmental Toxicology Program, Clemson University, Clemson, USA \\ ${ }^{2}$ Department of Environmental Engineering and Earth Sciences, Clemson University, Clemson, USA \\ ${ }^{3}$ Chiral Technologies Inc., West Chester, USA
}

Email address:

lc@clemson.edu (C. Lee)

${ }^{*}$ Corresponding author

To cite this article:

Prabha Ranasinghe, Christopher Olivares, William Champion Jr, Cindy Lee. Atropisomeric Separation of PCB-95 by HPLC. Science Journal of Chemistry. Vol. 7, No. 2, 2019, pp. 39-48. doi: 10.11648/j.sjc.20190702.12

Received: February 3, 2019; Accepted: March 20, 2019; Published: June 29, 2019

\begin{abstract}
Pentachlorobiphenyl (PCB-95) is an environmentally significant chiral PCB, of which enantioselective toxicity, biodegradation and chiral stability studies have been limited to date, as no commercially available enantiomers exist for PCB-95 and due to the lack of an efficient preparatory chiral separation method. A selective, sensitive, and rapid high-performance liquid chromatography with UV detection (HPLC-UV) method has been developed and validated for the chromatographic separation and quantitation of PCB-95 enantiomers. In this study, we resolved enantiomers of PCB-95 using a cellulose tris (4-methylbenzoate) Chiralcel OJ- $H$ column. After evaluating mobile phase compositions and temperatures, optimum separation and detection were obtained with isocratic $100 \%$ n-hexane as the mobile phase, a column temperature of $20^{\circ} \mathrm{C}$, a flow rate of $1 \mathrm{~mL} / \mathrm{min}$, and a detection wavelength of $280 \mathrm{~nm}$. The total run time was 8 minutes. Enantiomer purity was confirmed using enantioselective gas capillary chromatography-electron capture detection. The developed method was validated as per International Conference on Harmonization (ICH) guidelines with respect to limit of detection, limit of quantification, precision, linearity, robustness and ruggedness.
\end{abstract}

Keywords: Enantioselective Studies, 2, 2', 3, 5', 6-Pentachlorobiphenyl, Chiralcel OJ - $H$, Liquid Chromatography

\section{Introduction}

Despite sharing identical molecular formula and structure, enantiomers have different three-dimensional arrangement of chemical substituents at each of their chiral centers. Some molecules display axial-chirality and do not possess a chiral center. Instead, they have an axis with a set of substituents in a particular spatial arrangement leading to atropisomers, which are not superimposable. These enantiomers and atropisomers retain the same physicochemical properties but different biochemical properties that interact differently with macromolecules such as enzymes, receptors and transporters [1]. Therefore, the racemic mixtures and their individual stereoisomers can differ significantly in pharmacology, toxicology, pharmacokinetics and other biological processes [2]. Enantiomeric toxicity in the pharmaceutical industry has been greatly studied; and the US Food and Drug Administration (FDA) also recommended assessment of the enantiomeric activity of racemic drugs before they are released to the market [3]. However, there are several other sources such as agriculture and chemical industries that produce chiral compounds that are worth studying for their potential for enantioselectivity in biodegradation and toxicity for organisms and ecosystem health, especially if they are released to the environment in large quantities.

Polychlorinated biphenyls (PCBs) are a class of ubiquitous environmental pollutants that consist of chiral congeners and are of concern due to their persistent, bioaccumulative and toxic properties [4]. Before being banned in 1979 in the US, they were used as heat transfer fluids, hydraulic lubricants, dielectric fluids for transformers, capacitors, plasticizers, wax extenders, adhesives, organic diluents, deducting agents, pesticide extenders, cutting oils, carbonless reproducing papers and flame retardants $[5,6]$. Seventy-eight congeners of the 209 PCB congeners display axial chirality in their non-planar conformations [7]. However, only nineteen PCB congeners with three or four chlorine atoms exist as pairs of stable atropisomers [8] at ambient temperatures due to 
restricted rotation about the central $\mathrm{C}-\mathrm{C}$ biphenyl bond [9] Though they have being released to the environment as racemates, enantioselective enrichment of several of the stable atropisomeric PCBs in the environment have been documented [10-14].

However, lack of efficient chiral preparatory methods and unavailability of commercially available single enantiomers hinder enantioselective studies of chiral PCBs. As early as 1986, efforts to separate chiral PCBs were undertaken, but not all chiral PCBs were amenable to the methods $[15,16]$. Enantioselective degradation of PCB atropisomers have been studied in microcosms spiked with racemic mixtures of two chiral PCBs, PCB-132 and PCB-149 [17]. Authors observed enantioselective degradation for both congeners and these results point to the use of chiral analysis in understanding biotransformation mechanisms for PCBs in anaerobic environments. Aerobic enantioselective biodegradation of racemic mixtures of different $\mathrm{PCB}$ congeners has also been observed from microcosm experiments using different bacterial strains [18]. However, no data were found with spiking single enantiomers of chiral PCBs to compare the degradation rates of two enantiomers. Similarly, toxicology data for chiral PCBs are also scarce. However, there are a few studies conducted exposing organisms to single enantiomers of PCBs. For example, a toxicity study conducted using individual enantiomers of 2,2',3,4,6-pentachlorobiphenyl (PCB-88) and 2,2',3,3',4,4',6,6'-octachlorobiphenyl (PCB-197) reported that the $(+)$ enantiomers resulted in greater ethoxyresorufin-O-deethylase (EROD) activity compared to their (-) enantiomers when exposed to chick embryos [19]. Effects of racemic and single enantiomer toxicity of 2,2',3,3',6 pentachorbiphenyl (PCB -84) on $[3 \mathrm{H}]$-phorbol ester binding in rat cerebellar granule cells and ${ }^{45} \mathrm{Ca}^{2+-}$ uptake in rat cerebellum were also studied [20]. Observations suggested that each PCB-84 enantiomers can have different potencies, and these may differ from that of the racemic mixture [20]. These observations have important implications for understanding the mechanisms of neurotoxicity of chiral PCB congeners.

2, 2', 3, 5', 6-Pentachlorobiphenyl (PCB-95) (Figure 1) is one such chiral PCB congener that has been studied for neurotoxic effects [21-24]. However, enantioselective toxicity of PCB-95 is so far limited to two studies. A metabolomics study conducted using zebrafish embryos suggested that effects of single enantiomers of PCB-95 are more prominent than the racemates [24]. Another study was conducted to assess the effects of atropisomeric PCB -95 on ryanodine receptors (RyRs) and their influences on hippocampal neuronal networks [25]. The findings also revealed that the individual atropisomer had different potencies and also it differed from the racemate in enhancing $\left[{ }^{3} \mathrm{H}\right]$ ryanodine binding, microsomal $\mathrm{Ca}^{2+}$ transport and the $\mathrm{Ca}^{2+}$ dynamics in hippocampal neurons. Moreover, (+) PCB-95 plays a crucial role in enhancing the specific $\left[{ }^{3} \mathrm{H}\right]$ RyR binding. Rac- PCB-95 showed intermediate activity on enhancing $\left[{ }^{3} \mathrm{H}\right]$ RyR binding. In general, it is suggested that (-)-PCB-95, is more active towards the major isoforms; RyR1 and RyR2 than the $(+)-$
PCB-95 [25].
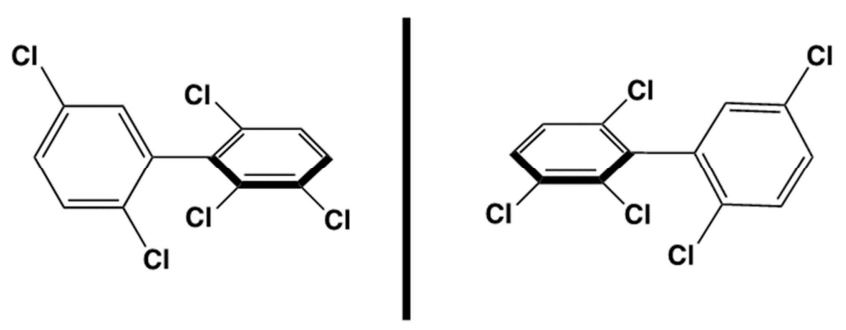

Figure 1. Structure of 2, 2, 3, 5, 6-pentachlorobiphenyl (PCB-95) enantiomers.

The two research groups that conducted enantioselective toxicity studies on PCB-95, both developed analytical methods to separate PCB-95 into its enantiomers using normal phase liquid chromatography. Feng et al. [25] separated PCB-95 atropisomers using, in series, three chiral columns composed of cellulose tris (4-methylbenzoate). However, limited information was provided on the chromatographic conditions. Xu et al. [24] separated PCB-95 enantiomers using a single column; Lux $5 \mu \mathrm{m}$ cellulose -3 column at $30^{\circ} \mathrm{C}$ with $100 \% \mathrm{n}$-hexane at $1 \mathrm{~mL} / \mathrm{min}$ [24]. Detailed information on run time and method validation was not easily accessible [26]. Therefore, there is still a need to provide a rapid, efficient and reliable method to separate PCB-95 atropisomers.

Chromatographic separation of enantiomers can be achieved either by forming diastereoisomers by chiral derivatization agents or by using a chiral stationary phase [27]. The use of chiral derivatization agent requires a functional group in the analyte such as an amine, hydroxyl, carboxyl, carbonyl etc. [3]. This requirement has been a disadvantage for neutral compounds such as PCBs; therefore, as an alternative to derivations, chiral stationary phases (CSPs) are used, which are robust and have gained considerable attention in the last few decades [28]. A CSP is composed of a chiral selector and a support (usually porous silica) and these selectors may be bonded to or coated on the support. Due to specific spatial constraints one of the enantiomers binds more strongly (ideal fit) than the other (non-ideal fit). The stereoisomer with the ideal fit interacts with the chiral stationary phase (CSP) via at least three interactions of contact, while the other interacts via two-sites or less leading to different retention times on the CSP [29-30]. Basically, the recognition mechanism on a chiral selector is based on a key-and-lock theory [31].

The chiral selectors used in CSPs include polysaccharide derivatives, cyclodextrin derivatives, macrocyclic antibiotics, proteins, ligand exchange complexes, crown ethers, imprinted polymers and some low-molecular-mass selectors such as Pirkle-type compounds [30]. Among them, cellulose, amylose ester and carbamate polymer derivatives coated onto a large pore silica backbone have proven to be most successful for chiral resolution using a range of mobile phases from polar to non-polar [28; 32-33]. Commercial columns containing these CSPs include Chiralcel OD [cellulose tri (3, 5 dimethylphenylcarbamate)], Chiralcel OJ [cellulose tri (4-methylbenzoate)], and Chiralpak $\mathrm{AD}$ [amylose tri 


\section{(3,5dimethylphenylcarbamate)] [27, 30].}

These polymers consist of a large number of functional groups that are able to bind with a wide range of substances through hydrogen bonding, $\pi-\pi$, dipole-dipole, and steric interactions [34]. Since there are no ionizable groups on the polysaccharide CSPs, neutral enantiomers can also be separated with polysaccharide CSPs [34]. PCBs are neutral compounds and 15 out of 19 chiral PCBs including PCB-84, PCB-91, PCB-95, PCB-131, PCB-132, PCB-135, PCB-136, PCB-139, PCB-149, PCB-171, PCB-174, PCB-175, PCB-176, PCB-196, and PCB-197 have been separated with reversed-phase supercritical fluid chromatography on a permethylated $\beta$-cyclodextrin column [35].

In this work, we report a rapid and reliable HPLC-based chiral resolution of the enantiomers of PCB-95 using a polysaccharide based CSP column, Chiralcel OJ- $H$, with short retention times. Use of HPLC based methods are required for the preparative chromatography that is necessary to conduct studies requiring single enantiomers for studying enantiomeric selectivity of biological processes such as toxicity, biodegradation, and biotransformation.

\section{Experimental Methods}

\subsection{Materials}

2, 2', 3, 5', 6-Pentachlorobiphenyl (PCB-95) (50/50 racemic mixture, purity $99.9 \%$ ) was purchased from Accu-Standard (New Haven, Cincinnati, USA). n-Hexane and isopropyl alcohol (IPA) were HPLC grade and purchased from Fisher Scientific (New Haven, Cincinnati, USA).

\subsection{HPLC}

Enantiomer separations were performed with a Thermo Ultimate 3000 high-performance liquid chromatograph coupled to a single wavelength UV detector (HPLC-UV) (Waltham, MA, USA). Chromeleon 6.8 was used to record and integrate peak areas.

\subsection{Chromatographic Conditions}

A Chiralcel OJ- $H$ (cellulose tris (4-methylbenzoate), $4.6 \mathrm{~mm} \times 250 \mathrm{~mm}, 5 \mu \mathrm{m}$,) column was utilized. Table 1 shows the mobile phase composition, flowrate, and temperature conditions tested. The UV detection occurred at $280 \mathrm{~nm}$ in all cases except for experimental condition 1 (Table 1, 100\% methanol), which had a detection wavelength of $210 \mathrm{~nm}$. The retention factor (k) between PCB-95 and the injection peak was determined as $k=\left(t_{R}-t_{0}\right) / t_{0}$, where $t_{R}$ and $t_{0}$ were the retention times of retained and unretained compounds, respectively. In this study $t_{0}$ was determined based on the void markers. The selectivity was calculated as $\alpha=k_{2} / k_{1}$, where $k_{1}$ denotes the retention factor of eluent 1 and $k_{2}$ is retention factor of eluent 2 .

\subsection{Standard Preparation}

A standard stock solution of $1 \mathrm{mg} / \mathrm{ml}$ of PCB-95 was prepared in $\mathrm{n}$ - hexane. A working solution of $100 \mathrm{mg} / \mathrm{L}$ was prepared.

\subsection{Method Validation}

Method validation techniques such as linearity, limit of detection (LOD), limit of quantification (LOQ), precision, robustness and ruggedness were applied in this study. Linearity was established by injecting racemic PCB -95 in triplicate in the concentration range of $0.1-100 \mathrm{mg} / \mathrm{L}$. Signal-to-noise $(\mathrm{S} / \mathrm{N})$ ratios of $3: 1$ and 10:1 were used to determine the detection and quantification limits, respectively. Precision was established by using four different concentrations lowest to highest $(1,10,50,100 \mathrm{mg} / \mathrm{mL})$ over three different days. Ruggedness (variation of the retention time and resolutions day to day) was also determined using the above method.

\subsection{Enantiomer Purity}

Enantiomeric purity was determined by injecting eluent 1 and eluent 2 collected from the HPLC into a 6850 Agilent capillary gas chromatograph (GC) coupled to a ${ }^{63} \mathrm{Ni}$-electron capture detector $(\mathrm{ECD})$. A Chirasil-Dex $30 \mathrm{~m}$ length $\times 0.25$ $\mathrm{mm}$ diameter capillary column with $0.25 \mu \mathrm{m}$ film thickness was utilized for the analysis. Details of the method can be found in a previous study [12]. The enantioselective separation quality $(\mathrm{T})$ was determined for the HPLC fractions. $\mathrm{T}$ is defined as a ratio of the difference between the top of the first eluent peak to the minimum between two peaks divided by the height of the first eluent peak [36]. Further, elution order of PCB-95 enantiomers in HPLC, was confirmed by measuring optical rotations of first and second eluents using polarimetry (MCP 500) at a wavelength of $589 \mathrm{~nm}$.

\section{Results and Discussion}

\subsection{Method Optimizations}

The enantiomer separation quality depends on a number of parameters that must be carefully optimized. The different chromatographic conditions utilized in the study are presented in Table 1. The first experimental condition 1 (100\% methanol) was adapted from [37], which used a maximum absorbance as $210 \mathrm{~nm}$. However, PCB-95 was not separated into its enantiomers using this method. To determine the appropriate wavelength, absorbance spectra were obtained using a UV-VIS spectrophotometer (Varian Cary 50). The maximum absorbance wavelength for PCB-95 was determined as 280 $\mathrm{nm}$ and was, therefore, kept constant for the rest of the trials.

Method optimization was obtained by modifying parameters such as column temperature, mobile phase, and flow rate. Column temperatures play a crucial role in separating enantiomers since the separation is driven by enthalpy [26]. Furthermore, in chromatography, selectivity is driven by thermodynamics $(\ln (\mathrm{K})=-\Delta \mathrm{H} / \mathrm{RT}+\Delta \mathrm{S} / \mathrm{R})$ but column efficiency and peak sharpness, are driven by kinetics, where $\Delta \mathrm{H}, \Delta \mathrm{S}, \mathrm{T}$ and $\mathrm{R}$ stands for change of enthalpy, change of entropy, temperature and gas constant respectively [38]. 
Resolution is a contest between improved $\Delta \mathrm{tR}$ at lower temperature and decreased peak width at higher temperature, which means lower temperature gives greater differences in $\mathrm{tR}$ and higher temperature gives sharper peaks. Therefore, temperature was varied between $20-25{ }^{\circ} \mathrm{C}$ knowing that the lower temperature is advantageous to separation. Flow rate and the viscosity of the mobile phase were also considered when setting temperature. If the mobile phase was more viscous, temperature was slightly increased for the slower flow rates (Table 1).

Table 1. Parameters optimized during the method development.

\begin{tabular}{llll}
\hline Mobile phase & Flow rate (mL/min) & Column temperature $\left({ }^{\circ} \mathbf{C}\right)$ & Wavelength $(\mathbf{n m})$ \\
\hline $100 \%$ Methanol & 1 & 20 & 210 \\
$75 \%$ Isopropyl alcohol (IPA) and 25\% Hexane & 0.3 & 25 & 280 \\
$50 \%$ IPA and 50\% Hexane & 0.5 & 25 & 280 \\
$20 \%$ IPA and $80 \%$ Hexane & 0.5 & 20 & 280 \\
$10 \%$ IPA and 90\% Hexane & 0.5 & 20 & 280 \\
$100 \%$ Hexane & 1 & 20 & 280 \\
\hline
\end{tabular}

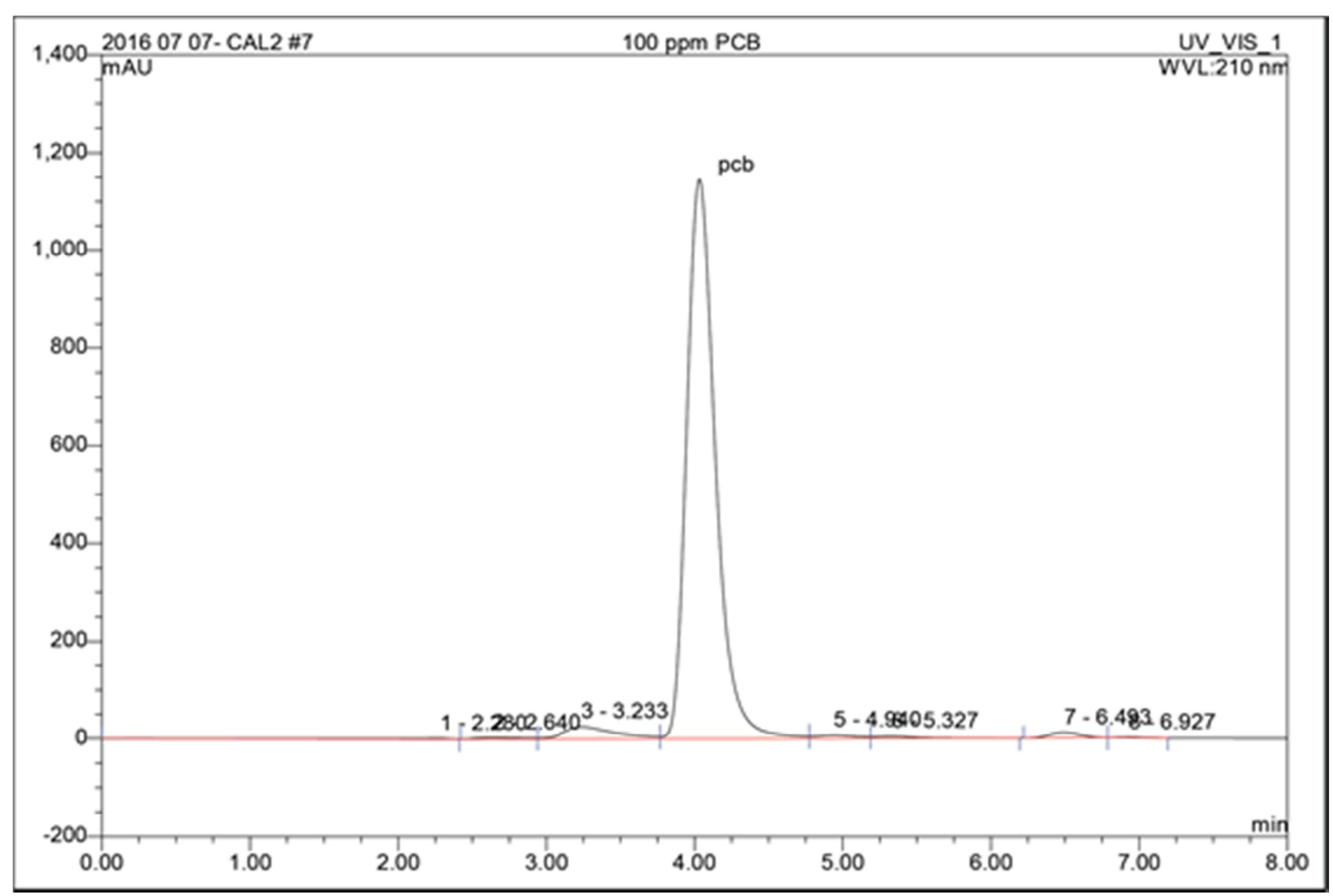

Figure 2. Mobile phase, 100\% Methanol. Single peak observed.

There is evidence of increasing separation efficiency of neutral compounds in normal phase liquid chromatography with the addition of a polar mobile phase additives [34]. This phenomenon can be explained as alcohol increase dipole-dipole interactions of the mobile phase with that of the CSP. The interaction allows the compound to be in the column for a longer time which in return gives better resolution. Therefore, methanol was used first as the mobile phase with a higher flow rate and comparatively lower temperature based on its low viscosity. Since no separation was observed (Figure 2), a combination of isopropanol alcohol (IPA) and hexane was evaluated. Better enantiomer separation of certain PCB methylsulfonyl metabolites was observed by Pham-Tuan et al. [36] when shifting the mobile phase from methanol to isopropanol. However, they did not observe complete baseline separation with IPA. Peak separation occurred (Figure 3) with this addition, but better resolution was observed by increasing the n-hexane proportion of the mobile phase (Figures 4-5). Similar results were observed by Champion et al. [39], when separating heptachlor, trans-chlordane and cis-chlordane using Chiralcel-OD columns. In the present study, maximum peak resolution was observed with 100\% n-hexane (Figure 6). 


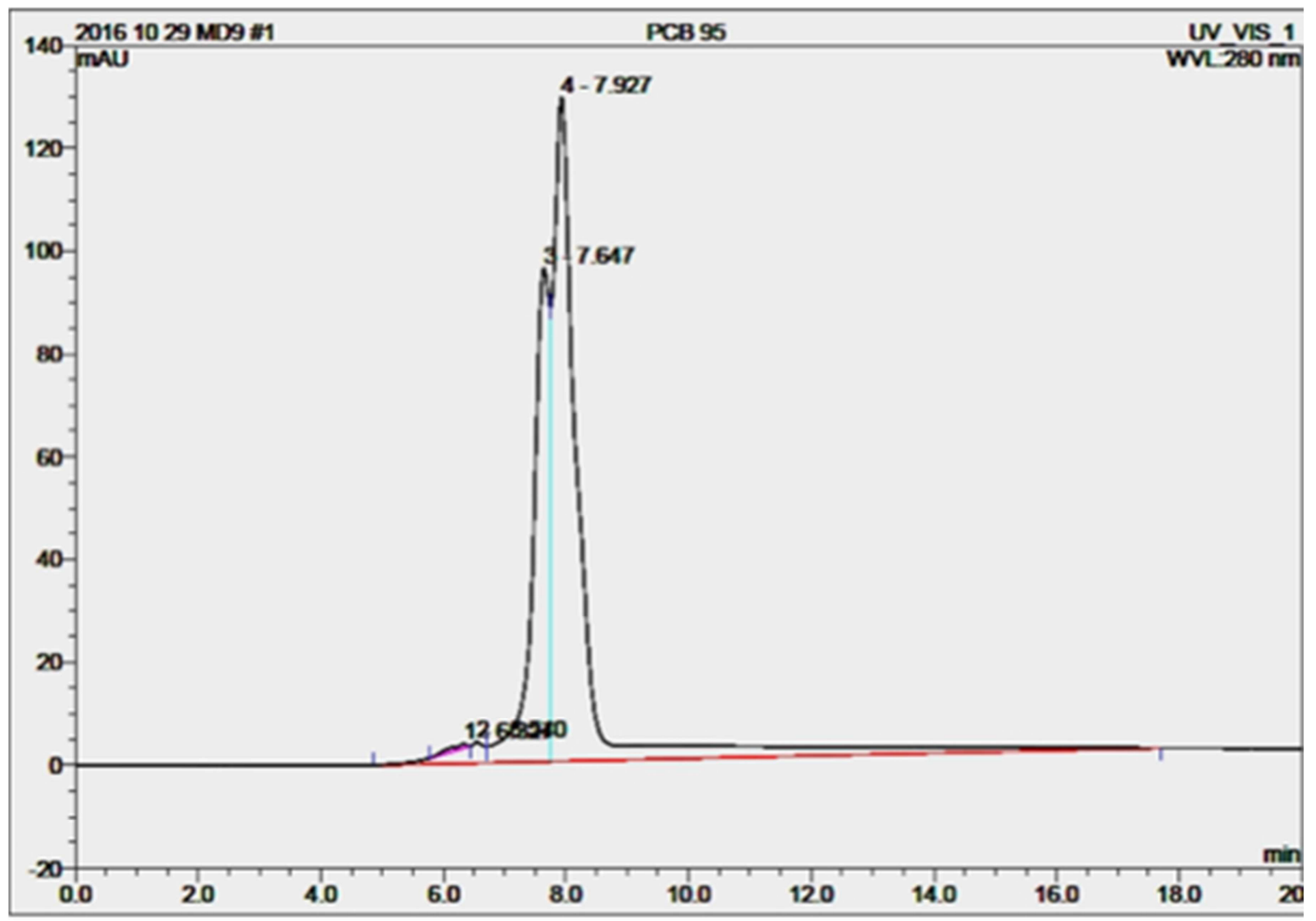

Figure 3. Mobile phase, 75\% isopropanol and 25\% n-hexane. Two peaks observed at 7.647 min and 7.927 min respectively. But peaks are with poor resolution.

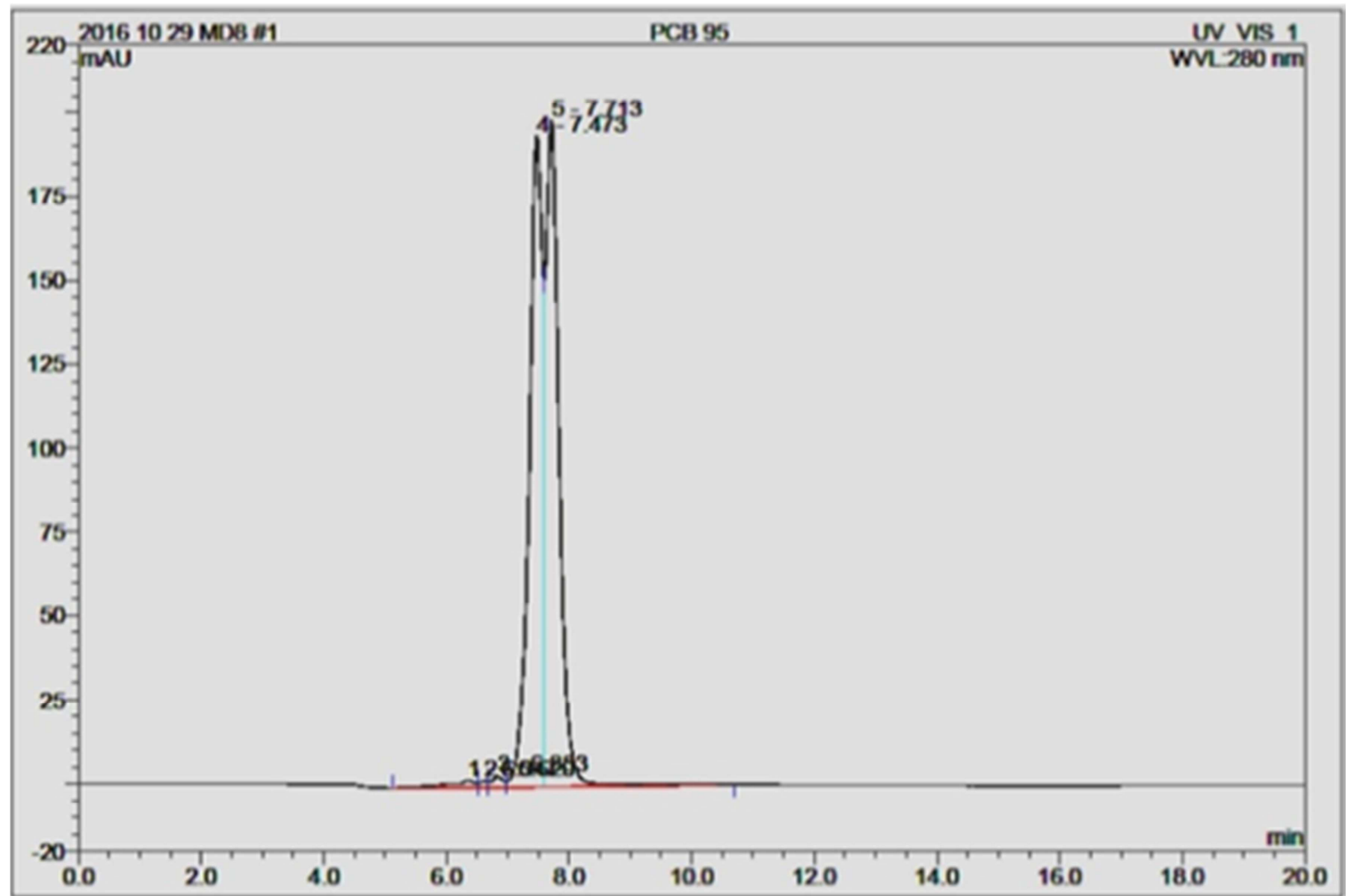

Figure 4. Mobile phase, 50\% isopropanol and 50\% n-hexane. Two peaks appeared at 7.473 min and 7.713 min respectively. However, peaks are still with poor resolution. 


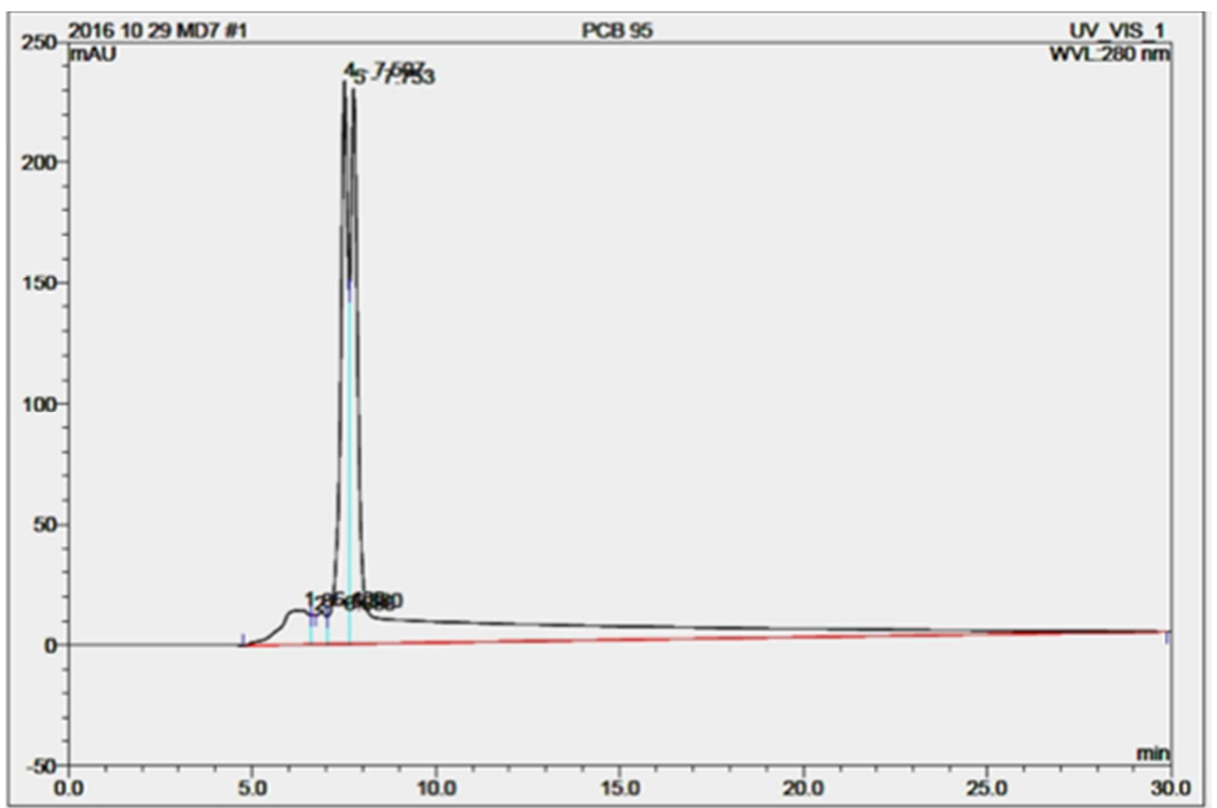

Figure 5. Mobile phase, 20\% isopropanol and $80 \%$-hexane. Two peaks appeared at 7.597 min and 7.753 min. Better separation was observed with increasing n-hexane percentage.

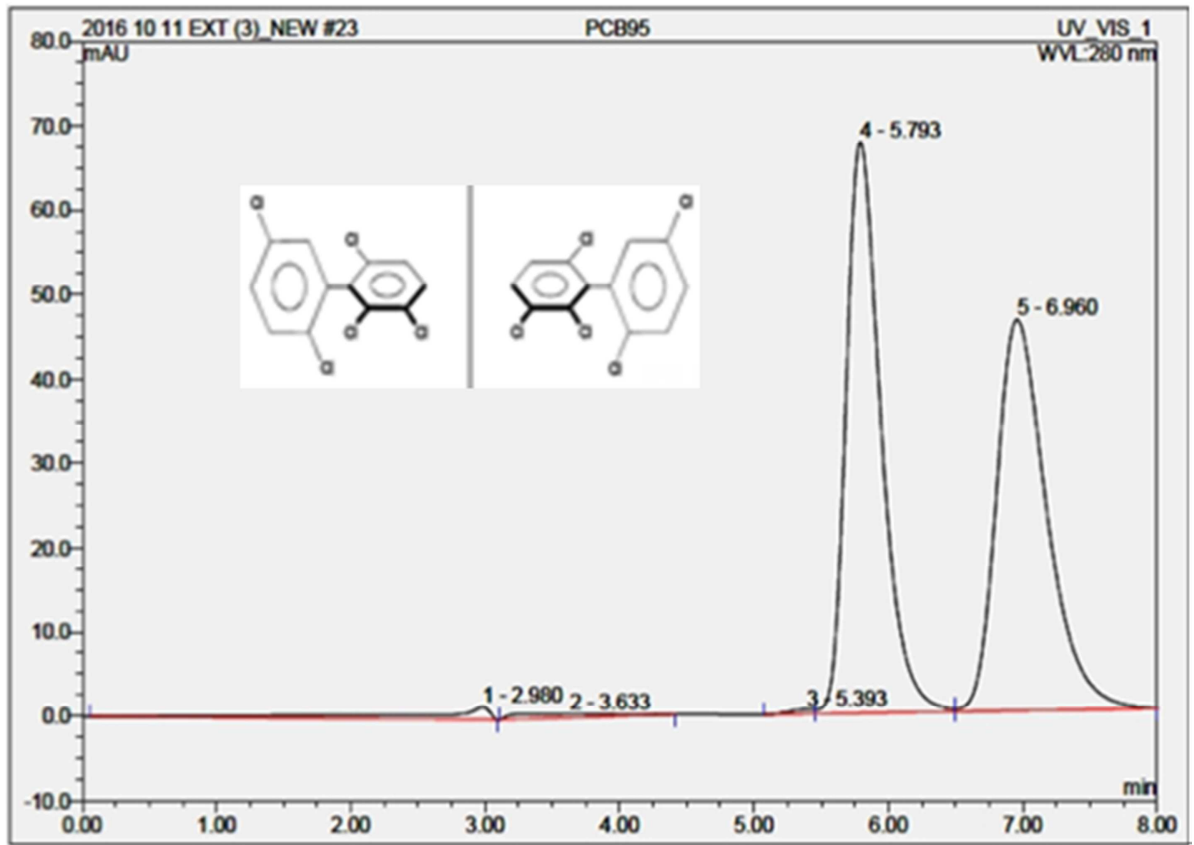

Figure 6. Mobile phase 100\% n-hexane. Fully resolved peaks observed at 5.793 min and 6.960 min respectively.

The retention factor $(k)$ for the eluent 1 and eluent 2 enantiomers of PCB-95 was 0.961 and 1.344, respectively, with $100 \%$ hexane. The selectivity $(\alpha)$ was 1.4 , which is satisfactory. All other mobile phase combinations resulted in selectivity around 1 , which indicates co-elution. The greater the selectivity value, the further apart the apices of the two peaks become. Shifting from the inclusion of a polar modifier to the completely non- polar mobile phase drastically increased the selectivity and the resolution for PCB-95.

The mechanism of the enantioseparation involves hydrogen bonding, $\pi-\pi$, dipole-dipole and inclusion in the chiral grooves [32]. The methylbenzoate polysaccharide stationary phase of the Chiracel OJ column forms hydrogen bonds with a polar mobile phase such as methanol [33]. In our study, the alcohols competed more effectively for the chiral solid phase than the neutral PCB-95 which resulted in poor resolution. In addition, we observed, increased enantiomeric resolution as the size of the alcohol increased because according to Wainer et al. [41] steric hindrance prevents large alcohols from occupying the stationary phase binding sites; therefore, the sites are more available for the analyte of interest.

\subsection{Method Validation}

The aim of an analytical method validation is to 
demonstrate that the analytical procedure is suitable for the intended purpose, which for this study was preparatory chromatography. However, to demonstrate the utility of the method, we also validated the method with respect to the range, linearity, LOD, LOQ as well as for its precision, robustness and ruggedness [42].

Calibration curves were constructed in the range of $0.1-$ $100 \mathrm{mg} / \mathrm{L}$ for racemic PCB-95. Linearity with regression coefficients, $\mathrm{R}^{2}$, of at least 0.99 was achieved. The regression equations for the first and second eluents were $\mathrm{y}=0.2312$ $\mathrm{X}+0.0131$ and $\mathrm{y}=0.2293 \mathrm{X}+0.0355$, respectively. It was also evident that the response was linearly related in the studied concentration range. As mentioned in the Experimental section, LOD was calculated with the $\mathrm{S} / \mathrm{N}$ ratio of 3 and was found to be $0.09 \mathrm{mg} / \mathrm{L}$ for eluent 1 and $0.08 \mathrm{mg} / \mathrm{L}$ for eluent 2 for PCB-95. LOQ was determined when the concentrations of analyte had a $\mathrm{S} / \mathrm{N}$ of 10 . The LOQ was 0.29 and $0.22 \mathrm{mg} / \mathrm{L}$ for

Table 2. Intraday and interday precision of the calibration curves for the analysis of PCB -95 enantiomers.

\begin{tabular}{|c|c|c|c|c|c|c|c|c|}
\hline \multirow{3}{*}{$\begin{array}{l}\text { Concentration } \\
(\mathrm{mg} / \mathrm{L})\end{array}$} & \multicolumn{4}{|c|}{ Intraday precision } & \multicolumn{4}{|c|}{ Interday precision } \\
\hline & \multicolumn{2}{|l|}{ Eluent 1} & \multicolumn{2}{|l|}{ Eluent 2} & \multicolumn{2}{|l|}{ Eluent 1} & \multicolumn{2}{|l|}{ Eluent 2} \\
\hline & Mean \pm SD & CV (\%) & Mean \pm SD & CV (\%) & Mean \pm SD & CV (\%) & Mean \pm SD & CV (\%) \\
\hline 0.5 & $0.108 \pm 0.001$ & 0.63 & $0.099 \pm 0.005$ & 5.30 & $0.108 \pm 0.000$ & 0.46 & $0.103 \pm 0.005$ & 5.15 \\
\hline 0.75 & $0.163 \pm 0.001$ & 0.47 & $0.150 \pm 0.006$ & 3.79 & $0.179 \pm 0.022$ & 12.42 & $0.157 \pm 0.010$ & 6.14 \\
\hline 1 & $0.212 \pm 0.002$ & 1.10 & $0.200 \pm 0.007$ & 3.36 & $0.221 \pm 0.009$ & 4.28 & $0.198 \pm 0.002$ & 1.09 \\
\hline 10 & $2.360 \pm 0.04$ & 0.19 & $2.400 \pm 0.007$ & 0.27 & $2.194 \pm 0.166$ & 7.54 & $2.434 \pm 0.049$ & 2.01 \\
\hline 25 & $5.924 \pm 0.017$ & 0.28 & $5.964 \pm 0.007$ & 0.11 & $6.025 \pm 0.142$ & 2.63 & $5.982 \pm 0.025$ & 0.426 \\
\hline 50 & $1.468 \pm 0.030$ & 0.26 & $11.626 \pm 0.101$ & 0.89 & $11.534 \pm 0.092$ & 0.8 & $11.443 \pm 0.024$ & 0.212 \\
\hline 100 & $23.155 \pm 0.062$ & 0.27 & $22.954 \pm 0.058$ & 0.25 & $22.459 \pm 0.985$ & 4.38 & $21.488 \pm 2.073$ & 9.647 \\
\hline
\end{tabular}

\subsection{Enantiomeric Purity}

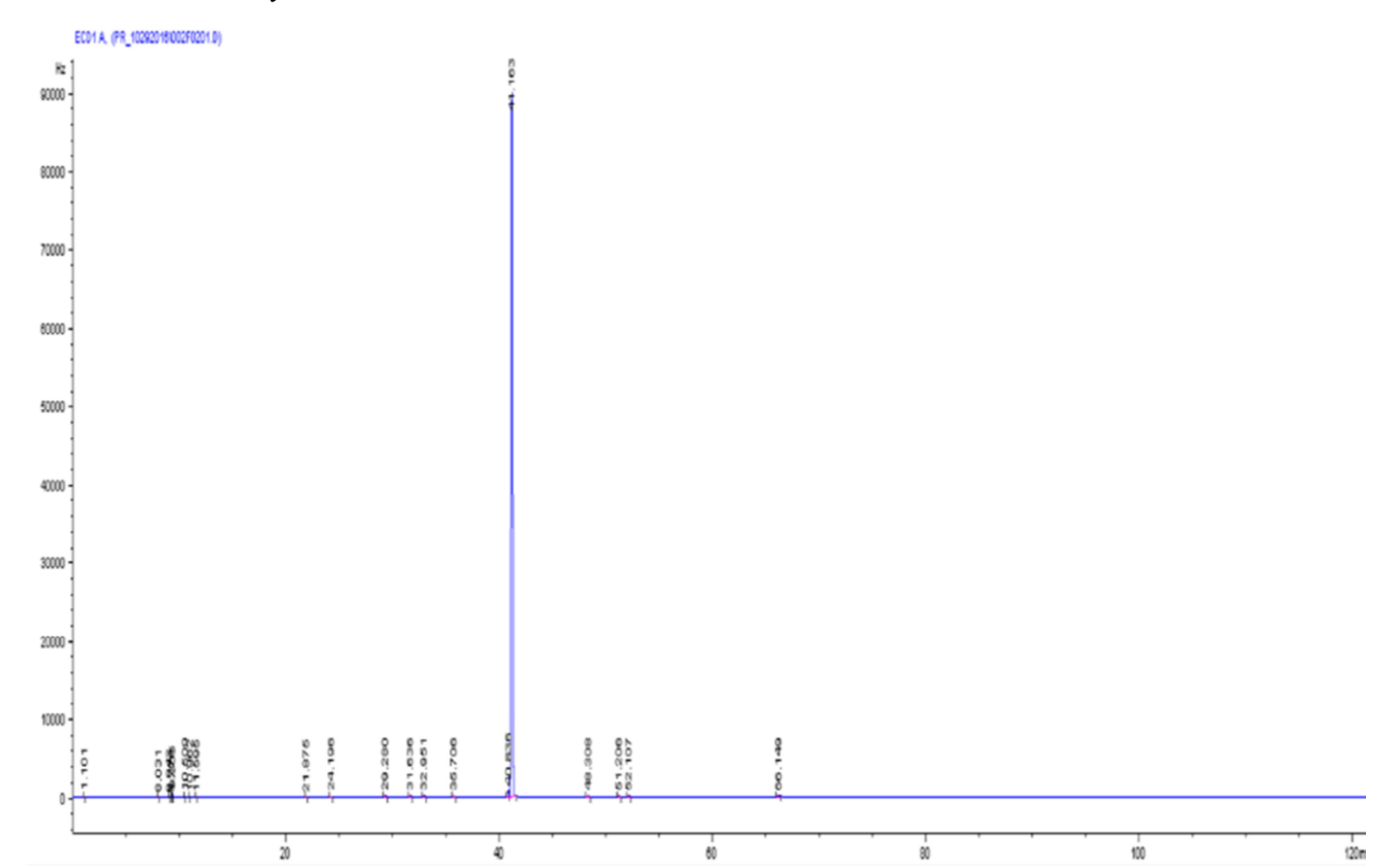

Figure 7. Eluent 1 of the HPLC had a retention time of 41.163 min in running in the capillary GC analysis. High purity $>98 \%$ was observed. See experiment

above for the experimental details. eluent 1 and eluent 2, respectively. Note that these values are much greater than those that can be determined using gas chromatography; therefore, this method is not ideal for quantification studies.

Table 2 provides data obtained for the precision. The coefficient of variance $(\mathrm{CV})$ for intra and inter day precision was less than $10 \%$ which suggests the method developed here is sufficiently precise. The $100 \mathrm{mg} / \mathrm{L}$ concentration resulted in a CV of $9.6 \%$ for eluent 2 and inter-day variation. All others were considerably less. The ruggedness (variation of the retention time day to day) was less than $1 \%$ for both enantiomers. The robustness of an analytical procedure measures its reliability of being unaffected by changes within a certain range [40, 43]. The results shown in Figures 2- 6 demonstrated the sensitivity of the method to different flow rates, composition of mobile phase and temperature. 
The configuration of chiral molecules can only be determined by using anomalous $\mathrm{X}$-ray diffraction and requires well-shaped single crystals. Other methods such as polarimetry, optical rotation dispersion (ORD), electronic circular dichroism (UV-CD) and vibration circular dichroism (VCD) are used but require comparisons to structural analogs, which are not always available [36]. In the current study, we utilized the polarimetry to assign absolute configurations. Polarimetric results revealed that the optical rotation of the separated enantiomers of PCB -95 was weak, however the first eluted peak of HPLC separation always displayed - rotation ($0.070 \pm 0.001)$ while the second eluted peak displayed + rotation $(+0.030 \pm 0.000)$ at the wavelength of $589 \mathrm{~nm}$.

Two separate peaks were obtained from GC-ECD analysis

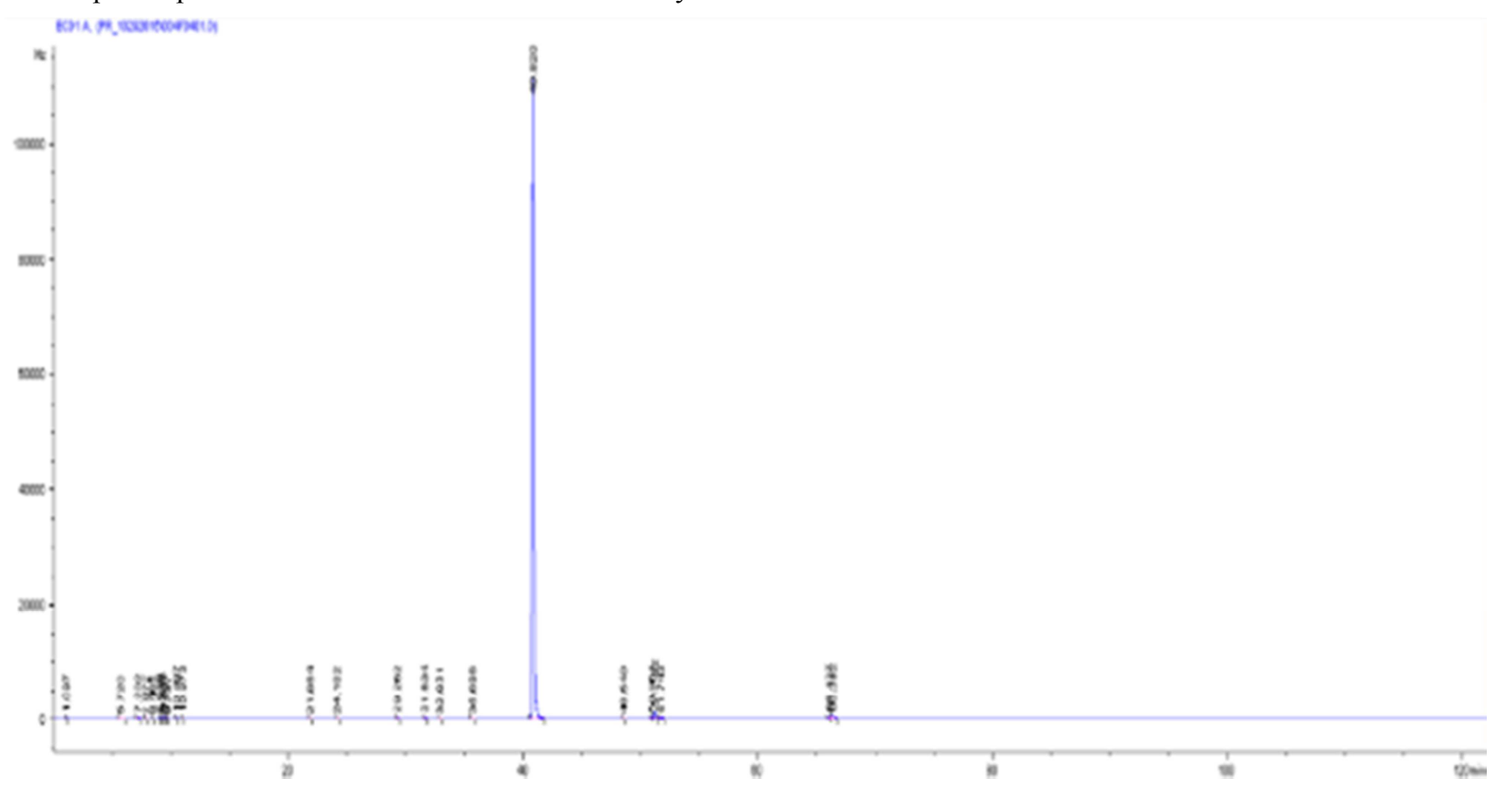

Figure 8. Enantiomeric separation of racemic PCB -95 with capillary gas chromatography. Peak 1 and peak 2 eluted at 40.835 and 41.158 minutes respectively. See experiment above for the experimental details.

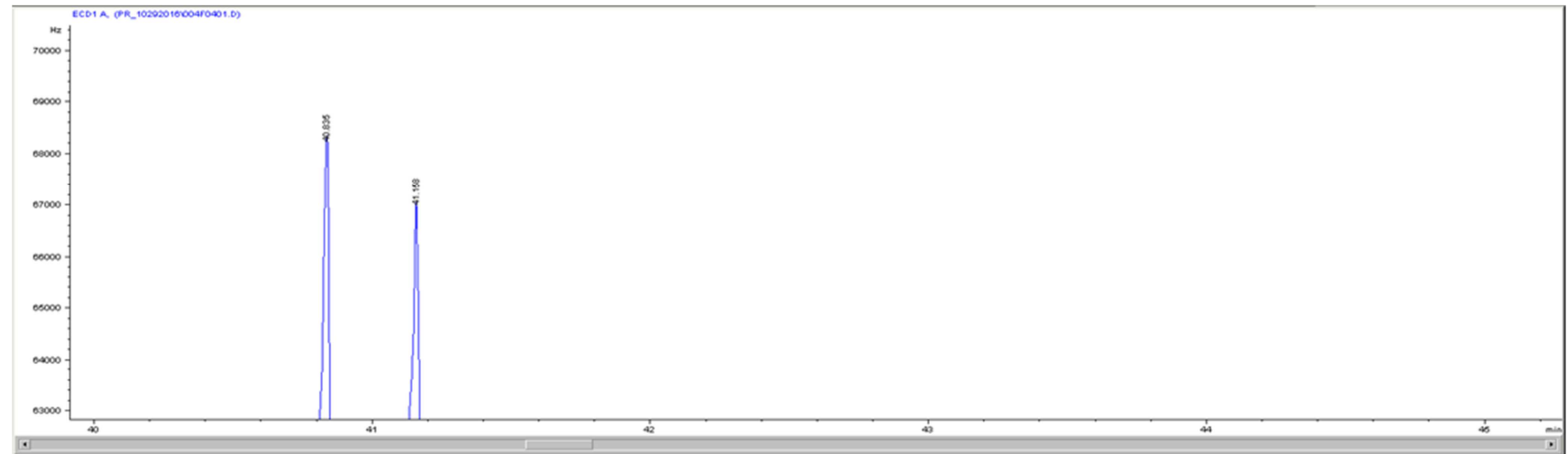

Figure 9. Enantiomeric separation of racemic PCB -95 with capillary gas chromatography. Peak 1 and peak 2 eluted at 40.835 and 41.158 minutes respectively. See experiment above for the experimental details.

\section{Conclusions}

Though, gas chromatography is the typically used for eluent 1 and eluent 2 collected from the HPLC using the optimized conditions. Retention times for eluent 1 and eluent 2 were $41.163 \mathrm{~min}$ and $40.820 \mathrm{~min}$, respectively (Figure 7-8). These retention times were further confirmed by injecting racemic $\mathrm{PCB}-95$ mixture and obtaining two peaks that corresponded with the eluent 1 and eluent 2 retention times (Figure 9). The enantioselective separation quality (T) for HPLC fractions was approximately 95\%. Determination of separation quality is important for the fraction collection process. In the ideal situation, a $\mathrm{T}=100 \%$ illustrates no co-elution occurred. With $\mathrm{T}=95 \%$, there is a small area of overlap that can be collected and run again for more complete separation. 
larger loading capacitates and shorter runtimes [39]. Therefore, a strategic approach to develop a LC chiral method for PCB -95 enantiomer separation using normal phase liquid chromatography with optimized chromatographic conditions was demonstrated and validated in the present study. The developed method is simple, reproducible, and sensitive and has the definite advantage of short run times and the use of only one column compared to other methods.

\section{Acknowledgements}

The authors are grateful for Mr. Kyle Martin from Furman University, South Carolina, Dr. April Hall of Nutra Manufacturing, and Mr. Jonathan Clayton, Clemson University, Clemson, SC for their support.

\section{References}

[1] S. W. Smith, Toxicol. Sci. 110, 4 (2009).

[2] S. Dai, C. S Wong, J. Qiu, M. Wang, T. Chai, L. Fang and S. Yang, J. Hazard. Mat. 280, 612 (2014).

[3] L. A. Nguye, H. He and C. Pham-Huy, Int. J. Biomed. Sci. 2, 85 (2006).

[4] L. J. Jackson and D. E Schindler, Environ. Sci. Technol. 30, 1861 (1996).

[5] J. L. Jacobson, S. W Jacobson and H. E. B. Humphrey, J. Pediatr. 116, 38 (1990).

[6] Agency for Toxic Substances and Disease Registry, Toxicological Profile for Polychlorinated Biphenyls (PCBs). (U.S. Department of Health and Human Services, Public Health Service, Atlanta, GA, 2000).

[7] G. P. Blanch, A. Glausch, V. Schurig, R. Serrano and M. Gonzale, J. Sep. Sci. 19, 392 (1996).

[8] H. J. Lehmler, S. J. Harrad, H. Hühnerfuss, I. Kania-Korwel, C. M. Lee, Z. Lu and C. S. Wong, Environ. Sci. Technol. 44, 2757 (2009).

[9] C. S. Wong, A. W. Garrison, P. D. Smith and W. T. Foreman, Environ. Sci. Technol. 35, 2448 (2001).

[10] L. Karasek., J. Hajslova, J. Rosmus and H. Huhnerfuss, Chemosphere 67, S22 (2007).

[11] A. Christoforidis, N. Stamatis, K. Schmieder and E. Tsachalidis, Chemosphere 70, 694 (2011).

[12] V. D. Dang, D. M. Walter and C. M. Lee, Environ. Sci. Technol. 44, $2836(2010)$.

[13] X. Wu, A. Pramanik, M. W. Duffel, E. G. Hrycay, S. M. Bandiera, H. J. Lehmler and I. Kania-Korwel, Chem. Res. Toxicol. 24, 2249 (2011).

[14] M. S. Ross, E. L. Pulster, M. B. Ejsmont, E. A. Chow, C. M. Hessel, K. A. Maruya and C. S Wong, Mar. Pollut. 63, 548 (2011).

[15] M. Püttmann, F. Oesch and L. W. Robertson, Chemosphere 15, 2061 (1986).
[16] P. Haglund, Chemosphere 32, 2133 (1996).

[17] U. Pakdeesusuk, W. J. Jones, C. M. Lee, A. W. Garrison, W. L. O'Niell, D. L. Freedman, J. T. Coates and C. S. Wong, Environ. Sci. Technol. 37, 1100 (2003).

[18] M. Moo-Young, Comprehensive Biotechnology, 2nd ed (Elsevier, 2011).

[19] L. E. Rodman, S. I. Shedlofsky, A. Mannschreck, M. Püttmann, A. T. Swim and L. W. Robertson, Biochem. Pharmacol. 41, 915 (1991).

[20] H. J. Lehmler, L. W. Robertsona, A. W. Garrison, P. R. Rao and S. Kodavanti, Toxicol. Lett., 156, 391 (2005).

[21] J. Gafni, P. W. Wong and I. N. Pessah, Toxicol. Sci. 77, 72 (2004).

[22] G. A Wayman, D. Yang, D. D Bose, A. Lesiak, V. Ledoux, D. Bruun, I. N. Pessah and P. J. Leine, Environ. Health Perspect. 120, 997 (2012).

[23] A. Lesiak, M. Zhu, H. Chen, S. M. Appleyard, S. Impey, P. J. Lein and G. A. Wayman, J. Neurosci. 34, 717 (2014).

[24] N. Xu, P. Mu, Z. Yin, Q. Jia, S. Yang, Y. Qian and J. Qiu, PLoS ONE 11, e0160584 (2016).

[25] W. Feng, J. Zheng, G. Robin, Y. Dong, M. Ichikawa, Y. Inoue, T. Mori, T. Nakano and I. N. Pessah, Environ. Sci. Technol. DOI: 10.1021/acs.est.7b04446, (2017).

[26] N. Xu, P. Mu, Q. Jia, T. Chai, Z. Yin, S. Yang and J. Qiu, Chinese J. Anal. Chem. 34, 795 (2015) [In Chinese].

[27] S. K. Maddi, S. Mohan, C. Ega, C. Rao, M. Yamasani and G. K. Scriba, J. Sep. Sci. 30, 1875 (2007).

[28] W. Lao and J. Gan, J. Chromatogr. A. 1117, 184 (2006).

[29] R. Bently, Arch. Biochem. Biophys. 414 (1), 1 (2003).

[30] M. Lämmerhofer, J. Chromatogr. A. 1217 (6), 814 (2010).

[31] H. Y. Aboul-Enein and I. Ali, Chiral Separations by Liquid Chromatography and Related Technologies (CRC Press, Boca Raton, FL, 2003).

[32] Y. Okamoto and T. Ikai., Chem. Soc. Rev. 37, 2593 (2008).

[33] J. Goossens, C. Foulon, A. L. Villard, J. Y. Puy, I. Lefebvre, P. Christian, C. Vaccher and J. P. Bonte, Biomed. Chromatogr. 19, 415 (2005).

[34] L. Zhou, C. Welch, C. Lee, X. Gong, V. Antonucci and Z. Ge, J. Pharm. Biomed. Anal. 49, 964 (2009).

[35] A. Zhang, W. Gao, B. Ma, L. Jin and C. Lin, Anal. Bioanal. Chem. 403, 2665 (2012).

[36] H. Pham - Tuan, C. Larsson, F. Hoffmann, A. Bergman, M. Fröba and H. Hühnerfuss Chirality 17, 266 (2005).

[37] S. Reich and V. Schurig, GIT Spezial. Sep. 16, 14 (1999) [In German].

[38] A. K. Badu-Tawiah (2010). Retrieved from fromhttps://knowledge.library.iup.edu/cgi/viewcontent.cgi?arti cle $=1928 \&$ context $=$ etd

[39] W. L. Champion, J. Lee, A. W. Garrison, J. C. DiMarco, A. Matabe, K. B. Prickett, J. Chromatogr. A. 1024 (2004). 
[40] G. K. Scriba, Chromatographia 75, 815 (2012).

[41] I. W. Wainer, M. C. Alembik and E. Smith, J. Chromatogr. A. 388, 65 (1987).

[42] International Conference on Harmonisation (ICH) Steering
Committee. ICH Q2B Validation of Analytical Procedures: Methodology. (CPMP/ICH/281/95) (European Agency for the Evaluation of Medicinal Products, International Commission on Harmonisation, London, 1996).

[43] D. Frühauf and M. Juz, J. Chromatogr. A. 1269, 242 (2012). 